\title{
Changes in subfoveal choroidal thickness after uncomplicated cataract surgery
}

\author{
Gaile Gudauskiene, Indre Matuleviciute, Ruta Mockute, Evelina Maciulaityte, Dalia Zaliuniene
}

\begin{abstract}
Background. Recent investigations show that phacoemulsification causing an inflammatory insult to the eye has an effect not only on retina but on the choroid as well. The purpose of this study was to evaluate the subfoveal choroidal thickness (SFCT) after uneventful phacoemulsification using swept-source optical coherence tomography (SS-OCT). Methods. This prospective study included 30 eyes of 23 patients with senile cataract undergoing uncomplicated phacoemulsification and intraocular lens implantation. SFCT and foveal retinal thickness (FRT) measurements were made at the same time, 1-2 PM preoperatively (P), 1 month (M1) and 3 months (M3) postoperatively using $1050 \mathrm{~nm}$ DRI Triton SS-OCT (Topcon, Tokyo, Japan). Postoperative changes in the SFCT, FRT and correlation of SFCT change with axial length, age, baseline intraocular pressure (IOP), IOP change were assessed.

Results. The mean SFCT increased statistically significantly 3 months after surgery in all sectors except the superior inner region. Of the factors affecting the SFCT, the change in the SFCT (M3/P), correlation with age and baseline IOP in almost all sectors was observed. The mean FRT increased significantly after the surgery in all sectors.

Conclusions. Insignificant subclinical increase in SFCT was observed 1 month after the cataract surgery. Significant increment in SFCT was detected 3 months postoperatively, which was correlated with surgery-induced IOP and ocular perfusion pressure change in the short term.
\end{abstract}

Key words: choroid, phacoemulsification, optical coherence tomography

Received: May 23, 2018; Accepted: December 5, 2018; Available online: December 18, 2018

https://doi.org/10.5507/bp.2018.076

Department of Ophthalmology, Lithuanian University of Health Sciences, Kaunas, Lithuania

Corresponding author: Indre Matuleviciute, e-mail: indrematule@yahoo.com

\section{INTRODUCTION}

The choroid is a vascular structure of the eye, supplying the outer retinal layers. The retina takes over $90 \%$ of the oxygen required from the choroid ${ }^{1}$. The choroid plays an important role in many ocular disorders such as central serous chorioretinopathy, choroidal neovascularization, polypoidal choroidal vasculopathy, high myopia, chorioretinal atrophy ${ }^{2}$. The choroidal structure is evaluated in clinical research to clarify the pathogenesis of these vision threatening disorders.

The visualisation of the choroid is complicated because of the retinal pigment epithelium (RPE) above and the underlying fibrous sclera. The analysis of the choroid using fluorescein angiography is limited by light absorption and scattering by the pigment of the RPE. The reflectivity of the choroid is difficult to distinguish from the surrounding structures using ultrasonography as well. Swept-source optical coherence tomography (SS-OCT) uses a longer wavelength than spectral domain OCT, penetrates tissues better and enables us to visualise the choroid $^{3}$. Choroidal changes are evaluated in various systemic ${ }^{4}$ and ocular ${ }^{5-11}$ disorders.

Cataract is responsible for $51 \%$ of world blindness, which represents about 20 million people (2010) (ref. ${ }^{12}$ ). This makes phacoemulsification the most frequent procedure performed in ophthalmology ${ }^{13}$. Phacoemulsification as a surgical procedure induces inflammation by stimulating the secretion of pro-inflammatory molecules such as interleukins, prostaglandins, vascular endothelial growth factors that may cause pseudophakic cystoid macular edema (CME) $\left(\right.$ ref. $\left.^{14,15}\right)$.

Recent investigations show that phacoemulsification has effect on the choroid as well. Some studies report that the choroidal thickness is increased after phacoemulsification $^{16-18}$ while others describe a tendency only ${ }^{19,20}$.

The purpose of our study was to evaluate the subfoveal choroidal thickness (SFCT) after uneventful phacoemulsification.

\section{MATERIAL AND METHODS}

This prospective research included consecutive patients who had uneventful phacoemulsification surgery for senile cataract performed at the Department of Ophthalmology of the Hospital of Lithuanian University of Health Sciences, Kaunas Clinics. Informed consent was obtained from all subjects before examination, Ethic Committee approved the study, the research followed the tenets of the Declaration of Helsinki.

Visually significant cataract was the inclusion criterion. Exclusion criteria were cataract precluding visualization of retinal fundus, high myopia, hyperopia, glaucoma, unstable fixation, retinal and vitreous pathology, traumatic cataract, hypertension, diabetes mellitus, any ocular surgery, corticosteroid therapy, increased (greater than $21 \mathrm{mmHg}$ ) postoperative intraocular pressure (IOP).

Cataracts were diagnosed on the basis of complete ophthalmologic examination by an experienced ophthal- 
mologist. The medical history of participants was collected. All eyes underwent detailed ophthalmic examination, including slit-lamp biomicroscopy, best-corrected visual acuity (BCVA), fundus examination, IOP measurement by Goldmann applanation tonometry, axial length (AL) measurement using Aladdin optical biometer based on optical low-coherence interferometry (Topcon, Tokyo, Japan).

\section{Cataract surgery}

Cataract surgeries were performed between November 2016 and April 2017 using a standard technique. Operations were performed under intravenous anesthesia. Phacoemulsification was carried out by one experienced surgeon using INFINITI ${ }^{\circledR}$ Vision System (Alcon Laboratories, Inc.). No intraoperative complications occurred. The acrylic intraocular lens was implanted "in the bag" in all operated eyes. All patients were prescribed dexamethasone eye drops (tapered weekly from 4 to 1 times per day), and ofloxacin eye drops ( 4 times daily for two weeks) postoperatively.

\section{SS-OCT}

As choroidal thickness demonstrates diurnal variation, measurements were performed at the same time 1-2 PM before (P), one (M1) and three months (M3) after cataract surgery. Trained examiners performed the procedure after pupil dilation. All patients were scheduled for a tomographic analysis of the whole foveal region with the SS-OCT (DRI OCT Triton; Topcon, Tokyo, Japan) at $1050 \mathrm{~nm}$ wavelength. Using this device, a retinal-choroidal map was produced according to the nine Early Treatment Diabetic Retinopathy Study (ETDRS) subfields using automated software in order to study different mean values of each sector. The mean thickness of each sector was automatically measured within 500 microns $(\mu \mathrm{m})$ from the center of the fovea in the central sector; $500-1500 \mu \mathrm{m}$ from the center of the fovea in the 4 juxtafoveal sectors (nasal, inferior, temporal, and superior), and 1500-3000 $\mu \mathrm{m}$ from the center of the fovea in the 4 extrafoveal sectors (nasal, inferior, temporal, and superior). The 9 automatically calculated ETDRS grid subfields were assessed: the SFCT and FRT in the central ring (C), nasal inner (iNAS), superior inner (iSUP), temporal inner (iTEM), inferior inner (iINF), nasal outer (oNAS), superior outer (oSUP), temporal outer (oTEM) and inferior outer (oINF) sectors. These scans were marked as the patient's baseline and were used referencing the subsequent scans using the "follow-up" function assuring us that the scans would be performed in the same position.

\section{Statistical analysis}

Statistical analysis was performed using SPSS Statistics for Windows, version 20.0 (IBM SPSS, Armonk, NY, USA). The results were presented as mean \pm standard deviation $(\mathrm{M} \pm \mathrm{SD})$. To evalute the changes in the SFCT and FRT the Wilcoxon Signed-Rank test and Friedman two way analysis were used. The factors affecting SFCT were evaluated using Spearman correlation. A $P$ value $\leq 0.05$ was considered statistically significant.

\section{RESULTS}

23 patients (30 eyes) were involved in the study [7 (30.4\%) males and 16 (69.6\%) females], 7 eyes were investigated only once after surgery, 3 eyes were investigated before surgery only. Mean age was $70.61 \pm 8.39$ years, (range: $57-84$ years). Mean P BCVA was 0.52 $\pm 0.21, \mathrm{M} 1$ BCVA - 0.96 \pm 0.19 , M3 BCVA - 0.95 \pm 0.21 . There was a statistically significant difference between the BCVA before and after surgery $(P<0.001)$. Mean AL was 23.32 \pm 0.88 $\mathrm{mm}$. The mean P IOP was $15.37 \pm 2.10 \mathrm{mmHg}$, M1 IOP $13.33 \pm 1.94 \mathrm{mmHg}$, M3 IOP - $12.57 \pm 2.56 \mathrm{mmHg}$. There was a statistically significant difference in IOP values before and after surgery $(P<0.001)$. CME was detected by SS-OCT in $1(3.7 \%)$ eye, 3 eyes were not investigated.

The mean FRT increased significantly after the surgery in all ETDRS sectors and did not differ between M1 and M3 in any sector (Table 1).

Table 1. The mean automatically measured FRT in EDTRS sectors.

\begin{tabular}{lccccccccc}
\hline & C & iSUP & iTEM & iINF & iNAS & oSUP & oTEM & oINF & oNAS \\
\hline Before surgery & & & & & & & & & \\
Mean $(\mu \mathrm{m})$ & 239.67 & 300.07 & 289.10 & 296.07 & 300.40 & 258.63 & 244.30 & 245.87 & 272.17 \\
SD & 20.89 & 14.30 & 16.10 & 16.72 & 13.79 & 12.03 & 18.57 & 15.80 & 14.29 \\
1 month after surgery & & & & & & & & \\
Mean $(\mu \mathrm{m})$ & 256.44 & 309.04 & 298.56 & 304.30 & 309.41 & 266.04 & 251.37 & 251.30 & 277.22 \\
SD & 44.08 & 15.18 & 20.28 & 18.54 & 16.00 & 13.69 & 16.14 & 16.68 & 13.74 \\
3 months after surgery & & & & & & & \\
Mean $(\mu \mathrm{m})$ & 252.64 & 308.23 & 293.32 & 302.73 & 309.45 & 267.32 & 250.77 & 251.73 & 273.18 \\
SD & 33.11 & 18.02 & 30.44 & 20.61 & 18.22 & 13.00 & 18.51 & 15.69 & 23.85 \\
$P^{*}$ & $<0.001$ & $<0.001$ & $<0.001$ & $<0.001$ & $<0.001$ & $<0.001$ & 0.001 & $<0.001$ & $<0.001$ \\
$P^{* *}$ & 0.001 & 0.001 & 0.001 & $<0.001$ & $<0.001$ & $<0.001$ & 0.01 & 0.001 & 0.001 \\
\hline
\end{tabular}

$P^{*}$ comparison between $\mathrm{P}$ and M1 FRT in 9 ETDRS sectors

$P^{* *}$ comparison between $\mathrm{P}$ and M3 preoperative and 3 months postoperative FRT in 9 ETDRS sectors

There was no statistically significant difference comparing M1 and M3 sectorial thickness. 
Table 2. The mean automatically measured SFCT in EDTRS sectors.

\begin{tabular}{|c|c|c|c|c|c|c|c|c|c|}
\hline & $\mathrm{C}$ & iSUP & iTEM & iINF & iNAS & oSUP & oTEM & oINF & oNAS \\
\hline \multicolumn{10}{|c|}{ Before surgery } \\
\hline \multirow[t]{2}{*}{ Mean $(\mu \mathrm{m})$} & 190.03 & 194.23 & 188.03 & 165.67 & 167.60 & 187.00 & 177.00 & 147.77 & 124.67 \\
\hline & 75.45 & 86.45 & 66.58 & 76.95 & 79.92 & 84.68 & 59.86 & 68.18 & 70.26 \\
\hline \multicolumn{10}{|c|}{1 month after surgery } \\
\hline Mean $(\mu \mathrm{m})$ & 192.56 & 188.85 & 190.56 & 171.59 & 169.74 & 184.15 & 179.63 & 152.33 & 124.78 \\
\hline SD & 83.50 & 84.25 & 73.03 & 79.20 & 84.96 & 86.77 & 65.80 & 68.70 & 72.64 \\
\hline \multicolumn{10}{|c|}{3 months after surgery } \\
\hline Mean $(\mu \mathrm{m})$ & 205.68 & 203.00 & 203.68 & 179.36 & 177.73 & 192.00 & 187.05 & 161.41 & 130.14 \\
\hline $\mathrm{SD}$ & 88.17 & 94.75 & 80.55 & 87.48 & 93.55 & 96.43 & 74.26 & 76.89 & 79.77 \\
\hline$P^{*}$ & $>0.05$ & $>0.05$ & $>0.05$ & 0.027 & $>0.05$ & $>0.05$ & $>0.05$ & 0.015 & $>0.05$ \\
\hline$P^{* *}$ & 0.035 & $>0.05$ & 0.010 & 0.022 & 0.039 & 0.022 & 0.044 & $<0.001$ & 0.042 \\
\hline$P^{* * *}$ & 0.015 & 0.041 & 0.014 & $>0.05$ & $>0.05$ & 0.010 & $>0.05$ & 0.031 & 0.042 \\
\hline$P^{* * * * *}$ & $>0.05$ & $>0.05$ & 0.003 & $>0.05$ & $>0.05$ & 0.025 & $>0.05$ & 0.001 & $>0.05$ \\
\hline
\end{tabular}

$P^{*}$ comparison between $\mathrm{P}$ and M1 SFCT in 9 ETDRS sectors

$P^{* *}$ comparison between $\mathrm{P}$ and M3 SFCT in 9 ETDRS sectors

$P^{* * *}$ comparison between M1 and M3 SFCT in 9 ETDRS sectors

$P^{* * * * *}$ comparison between $\mathrm{P}, \mathrm{M} 1$ and M3 SFCT in 9 ETDRS sectors

Table 3. Correlation between the difference of SFCT M1/M3 and P and various factors (correlation coefficient; significance level).

\begin{tabular}{|c|c|c|c|c|c|c|c|c|}
\hline & \multicolumn{2}{|c|}{ Age } & \multicolumn{2}{|c|}{$\mathrm{AL}$} & \multicolumn{2}{|c|}{ P IOP } & \multicolumn{2}{|c|}{ Change in IOP } \\
\hline & $\mathrm{R}$ & $P$ & $\mathrm{R}$ & $P$ & $\mathrm{R}$ & $P$ & $\mathrm{R}$ & $P$ \\
\hline \multicolumn{9}{|c|}{ Difference of SFCT 1 month after and before surgery } \\
\hline$\overline{\mathrm{C}}$ & -0.351 & 0.079 & 0.348 & 0.081 & 0.419 & 0.033 & -0.075 & 0.715 \\
\hline iSUP & -0.065 & 0.754 & 0.084 & 0.685 & 0.118 & 0.564 & 0.200 & 0.327 \\
\hline iTEM & -0.347 & 0.082 & 0.404 & 0.041 & 0.288 & 0.154 & -0.160 & 0.435 \\
\hline iINF & -0.244 & 0.230 & 0.561 & 0.003 & 0.309 & 0.125 & -0.177 & 0.387 \\
\hline iNAS & -0.450 & 0.021 & 0.313 & 0.119 & 0.413 & 0.036 & -0.205 & 0.315 \\
\hline oSUP & -0.572 & 0.002 & 0.423 & 0.031 & 0.431 & 0.028 & -0.187 & 0.360 \\
\hline oTEM & -0.256 & 0.207 & 0.331 & 0.099 & 0.150 & 0.464 & -0.104 & 0.612 \\
\hline oINF & -0.005 & 0.982 & 0.402 & 0.042 & 0.254 & 0.211 & -0.175 & 0.393 \\
\hline oNAS & -0.362 & 0.069 & 0.504 & 0.009 & 0.393 & 0.047 & -0.193 & 0.345 \\
\hline \multicolumn{9}{|c|}{ Difference of SFCT 3 months after and before surgery } \\
\hline $\mathrm{C}$ & -0.641 & 0.001 & 0.216 & 0.335 & 0.591 & 0.004 & -0.413 & 0.056 \\
\hline iSUP & -0.194 & 0.387 & 0.076 & 0.737 & 0.288 & 0.307 & -0.272 & 0.221 \\
\hline iTEM & -0.601 & 0.003 & 0.139 & 0.538 & 0.488 & 0.021 & -0.300 & 0.175 \\
\hline iINF & -0.519 & 0.013 & 0.117 & 0.605 & 0.512 & 0.015 & -0.600 & 0.003 \\
\hline iNAS & -0.535 & 0.010 & 0.184 & 0.412 & 0.579 & 0.005 & -0.494 & 0.019 \\
\hline oSUP & -0.656 & 0.001 & 0.062 & 0.784 & 0.585 & 0.004 & -0.305 & 0.168 \\
\hline oTEM & -0.692 & $<0.001$ & 0.361 & 0.099 & 0.660 & 0.001 & -0.277 & 0.212 \\
\hline oINF & -0.539 & 0.010 & 0.159 & 0.479 & 0.553 & 0.008 & -0.136 & 0.547 \\
\hline oNAS & -0.589 & 0.004 & 0.188 & 0.402 & 0.624 & 0.002 & -0.450 & 0.036 \\
\hline
\end{tabular}

The mean SFCT increased statistically significantly (M3/P) in all sectors except iSUP. Comparing all three visits, statistically significant increase was apparent in iTEM, oSUP, oINF sectors (Table 2).

Analyzing the SFCT affecting factors, the change in the SFCT (M1/P) correlated with age, AL and preoperative IOP in a few different sectors, no significant cor- relation with the change in IOP (M1/P) was observed. Evaluating the effect on the change in the SFCT (M3/P), correlation with age and preoperative IOP in almost all sectors was observed, while correlation with the change in IOP was observed in 3 out of 9 EDTRS sectors and there was no correlation with AL at all. Table 3 summarises all the correlations evaluated. 


\section{DISCUSSION}

Cataract surgery is the most frequent operation in ophthalmology which can cause an inflammatory insult to the eye. This study investigated the possibility that it may lead to an increase in the choroidal thickness at the posterior pole.

In our study (30 eyes) we found that FRT before surgery was $239.67 \pm 20.89 \mu \mathrm{m}, 1$ month after surgery $256 \pm 44.08 \mu \mathrm{m}, 3$ months after surgery $252 \pm 33.11 \mu \mathrm{m}$. There was a significant difference between FRT before and 1 month after surgery $(P<0.001)$. The mean FRT increased significantly after the surgery in all ETDRS sectors and did not differ between 1 and 3 months after surgery in any sector. Yasuo et al. found that the mean FRT of the 29 eyes at baseline and at postoperative 1 and 3 months was $222.7,230.8 \mathrm{~d} 225.9 \mu \mathrm{m}$, respectively. They observed a tendency of the increased FRT at 1 month postoperatively, although it did not reach the level of statistical significance ${ }^{21}$. The results showed that FRT before surgery was significantly lower comparing with the results 1 month after the surgery. These changes of thickness could be caused by inflammation after phacoemulsification, although all surgeries were uneventful. There are some studies, which compare postoperative effects after femtosecond laser cataract surgery (FLACS) and phacoemulsification. Ecsedy et al. analysed postoperative FRT and compared results between phacoemulsification and FLACS groups. 1 week after cataract surgery FRT in the phacoemulsification group was found to be significantly higher than in the FLACS group ${ }^{22}$.

Different studies analysed changes in SFCT after cataract surgery as the vascular choroid structure is the thickest at the macula ${ }^{23}$. In our research, the mean SFCT increased significantly 3 months after cataract surgery comparing with the results evaluated before the procedure $(205.68 \pm 88.17 \mu \mathrm{m}$ vs. $190.03 \pm 75.45 \mu \mathrm{m})$. Yilmaz et al. also studied changes in SFCT after phacoemulsification. Results of this research showed that SFCT was increased but did not bring out statistically significant difference between preoperative and postoperative SFCT (ref. ${ }^{24}$ ). Pierru et al. compared SFCT before and after uncomplicated phacoemulsification using enhanced depth imaging optical coherence tomography (EDI-OCT). They found that postoperative SFCT was thicker comparing with the preoperative results and especially for eyes with pseudophakic CME by 1 month $^{25}$. Asena et al. compared choroidal thickness alterations within the first month after FLACS and phacoemulsification. They found that there was no significant difference in SFCT before and after surgery in the FLACS patients $(P=0.37,0.10$, and 0.59$)$. Postoperative SFCT increased significantly compared with the preoperative SFCT and first day, one week, and one month after the procedure in the phacoemulsification group $\left(P=0.003,0.02\right.$, and 0.02 , respectively) $\left(\right.$ ref. $\left.{ }^{26}\right)$. It may also indicate the inflammatory effect of phacoemulsification.
Ohsugi et al. examined 100 eyes of Japanese patients after phacoemulsification. The increase in the mean SFCT was significant 3 days after surgery. There was a negative correlation between SFCT changes and postoperative IOP as well as $\mathrm{AL}\left(\right.$ ref $^{27}$ ). In our research, postoperative IOP was significantly reduced which leads to the increment in ocular perfusion pressure (OPP). This increase in OPP might also have been the reason for increased SFCT in the short term ${ }^{28}$.

All studied patients were prescribed anti-inflammatory dexamethasone eye drops one month postoperatively. This suppressive therapy reduces post-cataract inflammatory reaction and retinal inflammation ${ }^{29}$. We can assume that local hormone therapy could also diminish the inflammatory reaction in the choroid. However, the inflammatory mechanism alone was unlikely to cause morphologic choroidal changes after phacoemulsification in the long term as all patients were treated with anti-inflammatory therapy. Furthermore, in our study the increase of SFCT became significant when the hormone therapy was finished. Despite the postoperative inflammation mechanism, cataract surgery may induce release of prostaglandins which lead to an increase in uveoscleral outflow that might also contribute to the increase in SFCT. To sum up, different factors, including inflammation, increased OPP and uveoscleral outflow, activated metabolism, may be also responsible for the change in SFCT.

\section{Limitations}

The sample size might have affected the significance of the results. In addition, several patients refused to come to postoperative visits and did not complete the planned protocol. In addition, the balance between male and female participants was not reached. Another important limitation of this study is that we could not confirm when the increased SFCT could return to baseline. An extended investigation with a larger sample size and longer follow - up period could help to clarify our results.

\section{CONCLUSION}

Except for inferior sectors, SFCT changes were insignificant 1 month after the cataract surgery. However, there was a significant increase in SFCT 3 months after surgery in all sectors except iSUP. There was a significant negative correlation of the change in IOP M3/P and the SFCT changes at iINF, iNAS, oNAS regions, whereas change in IOP 1 month after surgery did not correlate with the SFCT changes. Phacoemulsification may induce SFCT thickening that correlates with postoperative changes of IOP and OPP in the short term. Subclinical increase in FRT was found. This decreased but did not reach preoperative values during follow-up. Further investigations and longer duration of follow-up should be continued to better clarify the effect of phacoemulsification on the choroid. 


\section{ABBREVIATION}

SFCT, Subfoveal choroidal thickness; SS-OCT, Sweptsource optical coherence tomography; FRT, Foveal retinal thickness; IOP, Intraocular pressure; RPE, Retinal pigment epithelium; CME, Cystoid macular edema; BCVA, Best-corrected visual acuity; AL, Axial length; P, Preoperative; M1, One month postoperative; M3, Three months postoperative; ETDRS, Early Treatment Diabetic Retinopathy Study; C, Central ring; iNAS, Nasal inner sector; iSUP, Superior inner sector; iTEM, Temporal inner sector; iINF, Inferior inner sector; oNAS, Nasal outer sector; oSUP, Superior outer sector; oTEM, Temporal outer sector; oINF, Inferior outer sector; M, Mean; SD, Standard deviation; FLACS, Femtosecond laser cataract surgery; OPP, Ocular perfusion pressure.

Author contributions: GG: main researcher, literature search, manuscript writing; IM: statistical analysis and literature search; RM, EM: literature search and manuscript writing; DZ: critical reading and cataract surgery. All authors: manuscript revision.

Conflict of interest statement: None declared.

\section{REFERENCES}

1. Nickla DL, Wallman J. The Multifunctional choroid. Prog Retin Eye Res 2010;29(2):144-68.

2. Gupta P, Cheung CY, Saw SM, Bhargava M, Tan CS, Tan M, Yang A, Tey F, Nah G, Zhao P, Wong TY, Cheng CY. Peripapillary choroidal thickness in young asians with high myopia. Invest Ophthalmol Vis Sci 2015; 56(3):1475-81.

3. Mrejen S, Spaide RF. Optical coherence tomography: imaging of the choroid and beyond. Surv Ophthalmol 2013;58(5):387-429.

4. Satue M, Obis J, Alarcia R, Orduna E, Rodrigo MJ, Vilades E, Gracia H, Otin S, Fuertes MI, Polo V, Larrosa JM, Pablo LE, GarciaMartin E. Retinal and Choroidal Changes in Patients with Parkinson's Disease Detected by Swept-Source Optical Coherence Tomography. Curr Eye Res 2018;43(1):109-15.

5. Ali Z, Shields CL, Jasani K, Aslam TM, Balaskas K. SweptSource Optical Coherence Tomography Angiography Findings in Torpedo Maculopathy. Ophthalmic Surg Lasers Imaging Retina 2017;48(11):932-5.

6. Lee SH, Lee EJ, Kim TW. Topographic correlation between juxtapapillary choroidal thickness and parapapillary deep-layer microvasculature dropout in primary open-angle glaucoma. $\mathrm{Br} \mathrm{J}$ Ophthalmol 2018;102(8):1134-40.

7. Ratra D, Tan R, Jaishankar D, Khandelwal N, Gupta A, Chhablani J, Agrawal R. Choroidal structural changes and vascularity index in stargardt disease on swept source optical coherence tomography. Retina 2018;38(12):2395-2400. doi: 10.1097/IAE.0000000000001879

8. Montorio D, Giuffrè C, Miserocchi E, Modorati G, Sacconi R, Mercuri S, Querques L, Querques G, Bandello F. Swept-source optical coherence tomography angiography in serpiginous choroiditis. Br J Ophthalmol 2018;102(7):991-5. doi: 10.1136/bjophthalmol-2017-310989

9. Jaisankar D, Raman R, Sharma HR, Khandelwal N, Bhende $M$, Agrawal R, Sudharshan S, Biswas J. Choroidal and Retinal Anatomical Responses Following Systemic Corticosteroid Therapy in Vogt-
Koyanagi-Harada Disease Using Swept-Source Optical Coherence Tomography. Ocul Immunol Inflamm 2017;12:1-9.

10. Garcia-Garcia O, Jordan-Cumplido S, Subira-Gonzalez O, Garcia-Bru P, Arias L, Caminal-Mitjana JM. Feasibility of swept-source OCT for active birdshot chorioretinopathy. Graefes Arch Clin Exp Ophthalmol 2017;255(8):1493-502.

11. Laíns I, Wang J, Providência J, Mach S, Gil P, Gil J, Marques M, Armstrong G, Garas S, Barreto P, Kim IK, Vavvas DG, Miller JW, Husain D, Silva R, Miller JB. Choroidal Changes Associated With Subretinal Drusenoid Deposits in Age-related Macular Degeneration Using Swept-source Optical Coherence Tomography. Am J Ophthalmol 2017;180:55-63.

12. The World Health Organization VISION 2020 Programs. Available from: URL: http://www.who.int/blindness/causes/priority/en/index1.html.

13. Gogate P. Comparison of various techniques for cataract surgery, their efficacy, safety, and cost. Oman J Ophthalmol 2010;3(3):105-6.

14. Wielders LHP, Schouten JSAG, Nuijts RMMA. Prevention of macular edema after cataract surgery. Curr Opin Ophthalmol 2018;29(1):4853.

15. Dong N, Xu B, Wang B, Chu L, Tang X. Aqueous cytokines as predictors of macular edema in patients with diabetes following uncomplicated phacoemulsification cataract surgery. Biomed Res Int 2015; 2015:126984.

16. Aslan Bayhan S, Bayhan HA, Muhafiz E, Kırboğa K, Gürdal C. Evaluation of choroidal thickness changes after phacoemulsification surgery. Clin Ophthalmol 2016;10:961-7.

17. Cheong KX, Tan CS. Long-term increase in subfoveal choroidal thickness after surgery for senile cataracts. Am J Ophthalmol 2015;159(3):608-9.

18. Uzun S, Pehlivan E. Evaluation of choroidal thickness changes after phacoemulsification surgery. Clin Ophthalmol 2016;10:1613-5.

19. Yılmaz T, Karci AA, Yilmaz I, Yılmaz A, Yıldırım Y, Sakalar YB. Long-Term Changes in Subfoveal Choroidal Thickness After Cataract Surgery. Med Sci Monit 2016;22:1566-70.

20. Jiang H, Li Z, Sun R, Liu D, Liu N. Subfoveal Choroidal and Macular Thickness Changes after Phacoemulsification Using Enhanced Depth Imaging Optical Coherence Tomography. Ophthalmic Res 2017 Oct 19. doi: 10.1159/000480240. [Epub ahead of print]

21. Yasuo N. Asako O, Taku T, Takashi U. Long-term increase in subfoveal choroidal thickness after surgery for senile cataracts. Am J Ophthalmol 2014;158(3):455-9.

22. Ecsedy M, Mihaltz K, Kovacs I, Takacs A, Filkorn T, Nagy ZZ. Effect of femtosecond laser cataract surgery on the macula. J Refract Surg 2011;27(10):717-22.

23. Margolis R, Spaide RF. A pilot study of enhanced depth imaging optical coherence tomography of the choroid in normal eyes. Am J Ophthalmol 2009;147(5):811-5.

24. Yilmaz T, Karci AA, Yilmaz I, Yilmaz A, Yildirim Y, Sakalar YB. Long-Term Changes in Subfoveal Choroidal Thickness After Cataract Surgery. Med Sci Monit 2016;22:1566-70.

25. Pierru A, Carles M, Gastaud P, Baillif S. Measurement of subfoveal choroidal thickness after cataract surgery in enhanced depth imaging optical coherence tomography. Invest Ophthalmol Vis Sci 2014;55(8):4967-74.

26. Asena BS, Karahan E, Kaskaloglu M. Retinal and choroidal thickness after femtosecond laser-assisted and standard phacoemulsification. Clin Ophthalmol 2017;11:1541-7.

27. Ohsugi H, Ikuno Y, Ohara Z. Changes in choroidal thickness after cataract surgery. J Cataract Refract Surg 2014;40:140-91.

28. Quaranta L, Katsanos A, Russo A, Riva I. 24-hour intraocular pressure and ocular perfusion pressure in glaucoma. Surv Ophthalmol 2013;58(1):26-41.

29. Laurell CG, Zetterström C. Effects of dexamethasone, diclofenac, or placebo on the inflammatory response after cataract surgery. $\mathrm{Br} \mathrm{J}$ Ophthalmol 2002; 86(12):1380-4. 